\title{
Resource Allocation for Intelligent Reflecting Surface Enabled Heterogeneous Networks
}

\author{
Yongjun Xu, Member, IEEE, Zhijin Qin, Member, IEEE, Yu Zhao, Student Member, IEEE, Guoquan Li, Member, \\ IEEE, Guan Gui, Senior Member, IEEE, and Hikmet Sari, Fellow, IEEE
}

\begin{abstract}
Intelligent reflecting surface (IRS)-enabled communication systems provide higher system capacity and spectral efficiency by reflecting the incident signals from transmitters in a low-cost passive reflecting way. However, it poses new challenges in resource allocation due to surrounding interference and phase shift, especially when IRS is employed in heterogeneous networks (HetNets). In this paper, a joint power allocation and phase shift optimization problem is studied for the downlink IRSenabled HetNet, in which the IRS is deployed to enhance the communications between small cell users (SCUs) and associated base station (BS). The signal-to-interference-plus-noise ratio (SINR) received at the SCU is maximized by jointly optimizing the transmit power of the small-cell BS and the phase shift of the IRS, subject to the constraints on the minimum SINR requirement of the macro-cell user (MCU) and the phase shift. Although the formulated problem is non-convex, we develop an optimal power allocation and the IRS's passive array coefficient solution for the single-user scenario. For the multiuser scenario, we propose an iterative algorithm to maximize the total rates of SCUs for obtaining a suboptimal solution by an alternating iteration manner, where the sum of multipleratio fractional programming problem is converted into a convex semidefinite program (SDP) problem. Simulation results show that the proposed algorithm significantly improves the achieved transmission rates of SCUs compared to the case without the IRS.
\end{abstract}

Index Terms-Heterogeneous network (HetNet), intelligent reflecting surface (IRS), phase shift, power allocation, resource allocation.

\section{INTRODUCTION}

I NTELLIGENT reflecting surface (IRS) consists of a large number of low-cost passive reflecting elements, which has been considered as a promising technology to enhance the spectrum and energy efficiency as well as to

This work was supported by the Project Funded by the National Science and Technology Major Project of the Ministry of Science and Technology of China under Grant TC190A3WZ-2, National Natural Science Foundation of China under Grant 61901228, the Jiangsu Specially Appointed Professor under Grant RK002STP16001, Innovation and Entrepreneurship of Jiangsu Highlevel Talent under Grants CZ0010617002 and CZ002SC19001, Summit of the Six Top Talents Program of Jiangsu under Grant XYDXX-010, 1311 Talen Plan of Nanjing University of Posts and Telecommunications. (Corresponding authors: Zhijin Qin; Guan Gui)

Y. Xu and G. Li are with the School of Communication and Information Engineering, Chongqing University of Posts and Telecommunications, Chongqing 400065, China. (e-mails: \{xuyj, ligq\}@ cqupt.edu.cn)

Z. Qin is with the School of Electric Engineering and Computer Science, Queen Mary University of London, London E1 4NS, UK. (e-mail: z.qin@qmul.ac.uk)

Y. Zhao, G. Gui, and H. Sari are with College of Telecommunications and Information Engineering, Nanjing University of Posts and Telecommunications, Nanjing 210003, China. (e-mails: \{1018010409, guiguan, hikmet\}@jupt.edu.cn) realize cost-efficient wireless networks by reconfiguring the wireless propagation environment [1]-[4]. Particularly, each IRS element can reflect and change the phase shift of the incident signal from the base station (BS) in a passive way [5]. This approach can achieve three-dimensional (3D) passive beamforming without the need of any transmit radio-frequency (RF) chain [6]. Since the IRS is connected with the BS through an IRS controller, the reflected signal propagation can be smartly configured to meet the communication requirements, i.e., maximizing the system capacity and enhancing transmission security.

\section{A. Related Work}

For the resource allocation (RA) problem in HetNets, the key issue is to determine power allocation and user association strategies for obtaining good system capacity and achieving interference management [7]-[10]. However, for the IRSbased HetNets, phase shift of reflecting elements at the IRS should be optimized for interference management and system capacity improvement. The RA problems for IRS-enabled wireless networks have attracted great attention, in which the major efforts have been put on enhancing system secrecy and spectrum efficiency. For the single-user IRS-based multipleinput single-output (MISO) system with single eavesdropper, transmit power minimization and secrecy rate maximization have been studied in [11] and [12], respectively. Extended to the multi-user scenario, the secrecy rate maximization under one eavesdropper and the max-min secrecy rate under multiple eavesdroppers have been discussed in [13] and [14], respectively.

In addition to RA for secrecy communications, spectrum efficiency and energy efficiency have been widely investigated for the IRS-enabled systems. Particularly, the spectrum efficiency of a point-to-point IRS-assisted MISO communication system is maximized by optimizing the beamformer at the access point (AP) and the phase shift of the IRS in [15]. For an IRS-based multiuser MISO system, the energy efficiency (EE) of users is maximized in [16] by using the alternating optimization method (AOM). In [17], the total transmit power at the AP has been minimized for an IRS-aided single-cell wireless system, in which one IRS is deployed to facilitate the transmissions between an AP equipped with multiple antennas and multiple users, each is equipped with a single antenna. The suboptimal transmit beamforming of the active antenna array at the AP and the reflect beamforming of passive phase shifters have been achieved by using the AOM. Moreover, 
due to the advantage of IRS in capacity improvement, it has also been adopted to enhance the performance of various communication systems, such as Terahertz communication system [18], simultaneous wireless information and power transfer (SWIPT) system [19], [20], and non-orthogonal multiple access (NOMA) system [21]-[23]. The summary of existing work and comparison of the problem formulation along with solution domains is provided in Table I.

\section{B. Motivation and Contributions}

It is well-known that HetNets can obviously improve system coverage and network capacity gain by deploying small cells underlying the macrocell network [24]-[26]. The power allocation and beamforming design at the small cell BS (SBS) is applicable to improve the communication quality of indoor users and suppress the mutual interference or inter-cell interference (ICI) [27], [28]. However, the quality of service (QoS) of small-cell users (SCUs) can be heavily degraded by interior walls or strong fading channels, such as non-line of sight (NLOS). The IRS can be placed in such scenarios for improving the wireless signal strength. Hence, it is strongly desirable to explore the use of IRS to provide additional paths and construct stronger combined channels by intelligently adjusting the phase shifts of the passive IRS elements.

Motivated by the above, in this paper, we consider the downlink transmissions in an IRS-enabled HetNet where an SBS overlaying in the macrocell transmits signals to multiple SCUs and the IRS. The performance improvement of the single-antenna SCU is achieved through the IRS that forwards a suitable phase-shifted version of the transmitted signal from the SBS. To the best of our knowledge, the RA for such a system is still in its infancy. The contributions of this paper are summarized as follows:

- We investigate an IRS-enabled HetNet with two tiers where a single-antenna SBS transmits superposed signals to multiple single-antenna SCUs with the assistance of an IRS. For the single SCU scenario, the signal-tointerference-plus-noise ratio (SINR) received at the SCU is maximized by jointly optimizing the transmit power of the SBS and the phase shifts of IRS elements under the SINR constraint of the macrocell user (MCU) and the phase shift constraint. An Dinkelbach-based optimal iteration algorithm is proposed to solve the problem.

- For the multi-user scenario, the sum rate of SCUs is maximized by jointly optimizing the transmit power and phase shift. To tackle the coupled variables in the objective function and constraints, we propose an efficient algorithm based on the semidefinite programming (SDP) technique and successive convex approximation (SCA) to obtain a suboptimal solution in an alternating iteration manner.

- Simulation results show that IRS can improve the transmission rate of SCUs significantly, even for the scenario where the direct links of users are weak.

The rest of this paper is organized as follows. In Section II, the system model of the IRS-enabled HetNet is described and the RA problem for the single-user scenario is formulated and

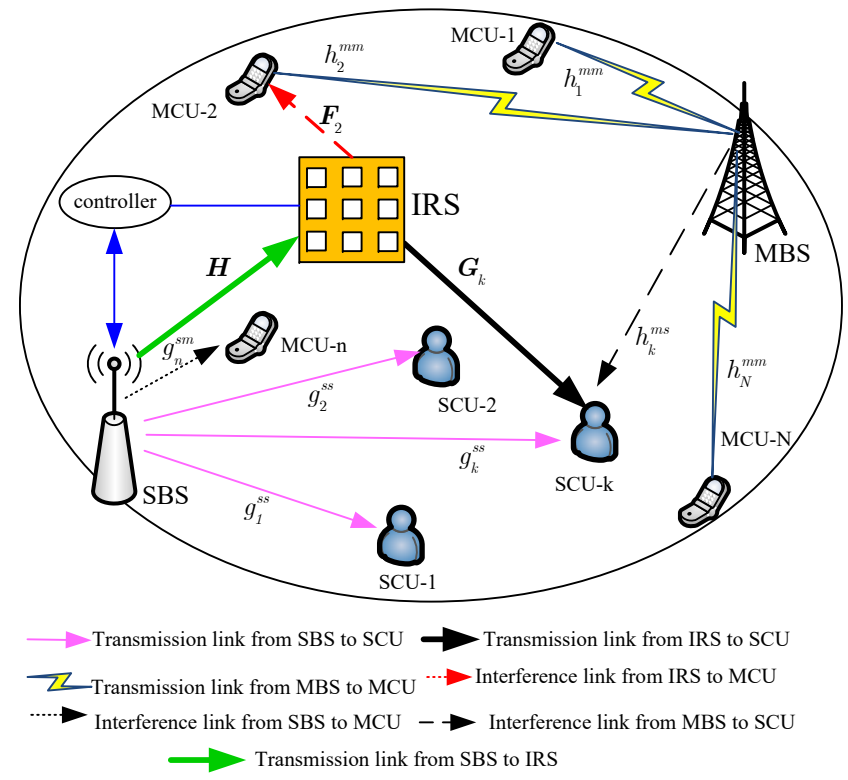

Fig. 1. An IRS-enhanced heterogeneous wireless network.

solved optimally. Section III formulates the RA problem for the multi-user case and provides a suboptimal solution. Section IV presents simulation results to show the effectiveness of the proposed algorithms. Finally, the concludes and future research direction are drawn in Section V.

\section{System Model and Single-User Resource ALLOCATION}

\section{A. System Model}

We consider the downlink transmission of the IRS-enabled HetNet, where an IRS is employed to enhance the transmission performance between the SBS and SCUs, as illustrated in Fig. 1. It is assumed that the IRS comprises $M$ passive reflecting units, denoted as $\mathcal{M}=\{1,2, \cdots, M\}, \forall m \in \mathcal{M}$, and is connected to a controller, which adjusts the IRS pattern for the desired signal reflection. A separate wireless control link serves for information exchange between the IRS controller and the SBS on the channel state information (CSI) and other information needed for implementing the joint design of the SBS transmit power and IRS reflection with the QoS guaranteed for the MCU [20]. We assume that the CSI is available at the BS via channel training or feedback from the users and the IRS [11], [12].

Define $\boldsymbol{H}=\left[h_{1}, h_{2}, \cdots, h_{M}\right]^{T} \in \mathcal{C}^{M}$ and $\boldsymbol{G}=$ $\left[g_{1}, g_{2}, \cdots, g_{M}\right]^{T} \in \mathcal{C}^{M}$ as the channel gain from the SBS to the IRS and the IRS to the SCU, and denote $h^{m m}$ and $h^{m s}$ as the channel gain from the MBS to the MCU and the MBS to the SCU. Define $g^{s m}$ and $g^{s s}$ as the channel gain from the SBS to the MCU and the SBS to the SCU. Define $\boldsymbol{F}=\left[f_{1}, f_{2}, \cdots, f_{M}\right]^{T} \in \mathcal{C}^{M}$ as the channel gain from the IRS to the MCU. Define $\phi=\left[\phi_{1}, \phi_{2}, \cdots, \phi_{M}\right]^{H}$ as the IRS reflection coefficients, where each $\phi_{m}=\beta_{m} e^{j \theta_{m}}$ comprises an amplitude coefficient $\beta_{m} \in[0,1]$ and a phase shift $\theta_{m} \in[0,2 \pi)$ of the $m$-th reflecting element, and $\left|\phi_{m}\right|=1$. Define $\boldsymbol{\Phi}=\operatorname{diag}(\boldsymbol{\phi}) \in \mathcal{C}^{M \times M}$ as the reflection 
TABLE I

SUMMARY OF RA IN IRS-BASED SYTEMS.

\begin{tabular}{|c|c|c|c|c|c|c|}
\hline Ref. & Type & Network Scenario & Problem & Interference Type & Optimal variable & Solution \\
\hline [17] & single-tier & multiuser IRS-based MISO system & min: total transmit power & inter-cell interference & beamforming and $\mathrm{RC}$ & suboptimal, closed-form \\
\hline [15] & single-tier & single-user IRS-based MISO system & max: data rate & inter-cell interference & beamforming and $\mathrm{RC}$ & suboptimal, no close-form \\
\hline$[16]$ & single-tier & multiuser IRS-based MISO system & max: EE & inter-cell interference & power allocation and $\mathrm{RC}$ & suboptimal, no close-form \\
\hline [18] & single-tier & multiuser IRS-based MISO system & max: sum rate & inter-cell interference & beamforming and $\mathrm{RC}$ & suboptimal, no close-form \\
\hline [19] & single-tier & multiuser MISO IRS-SWIPT system & min: total transmit power & Inter-cell interference & beamforming and $\mathrm{RC}$ & suboptimal, close-form \\
\hline [20] & single-tier & multiuser MIMO IRS-SWIPT system & max: weighted sum rate & inter-cell interference & beamforming and $\mathrm{RC}$ & suboptimal, close-form \\
\hline [21] & single-tier & multiuser SISO IRS-NOMA system & max-min: SINR & inter-cell interference & power allocation and $\mathrm{RC}$ & suboptimal, no close-form \\
\hline [22] & single-tier & multiuser MISO IRS-NOMA system & max: sum rate & inter-cell interference & beamforming and $\mathrm{RC}$ & suboptimal, no close-form \\
\hline [23] & single-tier & multiuser MISO IRS-NOMA system & min: total transmit power & inter-cell interference & beamforming and $\mathrm{RC}$ & suboptimal, no close-form \\
\hline $\begin{array}{l}\text { in this } \\
\text { paper }\end{array}$ & two-tier & multiuser IRS-based HetNets & max: sum rate & $\begin{array}{l}\text { inter-cell interference, } \\
\text { cross-tier interference }\end{array}$ & power allocation and RC & $\begin{array}{l}\text { optimal and suboptimal, } \\
\text { closed-form }\end{array}$ \\
\hline
\end{tabular}

coefficient matrix of the IRS. Since we aim to obtain the maximum designed signal, therefore, the amplitude coefficient is set as $\beta_{m}=1$ for simplicity [17]. As a result, we only need to optimize the phase shift of each IRS element. We denote $P$ and $p$ as the transmit power at the MBS and the SBS, respectively. Correspondingly, the SINRs received at the SCU and the MCU are

$$
\gamma^{\mathrm{SCU}}=\frac{p\left|\boldsymbol{G}^{H} \boldsymbol{\Phi} \boldsymbol{H}+g^{s s}\right|^{2}}{P h^{m s}+\sigma^{2}},
$$

and

$$
\gamma^{\mathrm{MCU}}=\frac{P h^{m m}}{p\left|g^{s m}+\boldsymbol{F}^{H} \boldsymbol{\Phi} \boldsymbol{H}\right|^{2}+\sigma^{2}} .
$$

where $(\cdot)^{H}$ denotes the conjugate transpose of a vector.

\section{B. Single-user Resource Allocation Algorithm}

In the IRS-enabled HetNet, we improve the QoS of SCU by adjusting the transmit power, meanwhile, the QoS of the $\mathrm{MCU}$ needs to be guaranteed. Since the data rate of user is in proportion to the SINR. Thus, the max-SINR of SCU with the MCU's QoS constraint is given by

$$
\text { P1 } \begin{aligned}
& \max _{p, \theta_{m}} \gamma^{\mathrm{SCU}} \\
& \text { s.t. } C_{1}: \gamma^{\mathrm{MCU}} \geq \gamma^{\min }, \\
& C_{2}: 0 \leq \theta_{m} \leq 2 \pi, \forall m, \\
& C_{3}: 0 \leq p \leq p^{\max },
\end{aligned}
$$

where $C_{1}$ denotes the QoS required by the MCU under the interference from the IRS and the SBS, $\gamma^{\min }$ represents the SINR threshold of the MCU, $C_{2}$ denotes the phase shift constraint at the IRS, $C_{3}$ denotes the transmit power range of the SBS, and $p^{\max }$ is the maximum transmit power at the SBS. Since we focus on finding the optimal power $p$ and phase shift $\theta_{m}$, the maximum transmit power limit at the MBS is omitted here.

According to $C_{1}$, we have the upper bound of $p$, that is

$$
p \leq \frac{P h^{m m}-\gamma^{\min } \sigma^{2}}{\gamma^{\min }\left|g^{s m}+\boldsymbol{F}^{H} \mathbf{\Phi} \boldsymbol{H}\right|^{2}} .
$$

According to the monotonicity of $p$, we have the optimal power allocation strategy

$$
p^{*}=\min \left(p^{\max }, \frac{P h^{m m}-\gamma^{\min } \sigma^{2}}{\gamma^{\min }\left|g^{s m}+\boldsymbol{F}^{H} \mathbf{\Phi} \boldsymbol{H}\right|^{2}}\right) .
$$

By substituting the optimal $p^{*}$ in (4) into the problem P1, we have the following optimization problem, i.e.,

$$
\begin{aligned}
& \mathbf{P 2} \quad \max _{\theta_{m}} \frac{A\left|\boldsymbol{G}^{H} \boldsymbol{\Phi} \boldsymbol{H}+g^{s s}\right|^{2}}{\left|g^{s m}+\boldsymbol{F}^{H} \boldsymbol{\Phi} \boldsymbol{H}\right|^{2}} \\
& \text { s.t. } C 2: 0 \leq \theta_{m} \leq 2 \pi, \forall m,
\end{aligned}
$$

where $A=\frac{P h^{m m}-\gamma^{\min } \sigma^{2}}{\gamma^{\min }\left(P h^{m s}+\sigma^{2}\right)}$ if $p^{*}<p^{\max }$, otherwise $A=p^{\max }$. It is a fractional form which belongs to a nonlinear fractional program [29]. Without loss of generality, we define the optimal objective function as

$$
\lambda^{*}=\frac{A\left|\boldsymbol{G}^{H} \mathbf{\Phi} \boldsymbol{H}+g^{s s}\right|^{2}}{\left|g^{s m}+\boldsymbol{F}^{H} \mathbf{\Phi} \boldsymbol{H}\right|^{2}} .
$$

We are now ready to introduce the following Theorem.

Theorem 1: The maximum objective value $\lambda^{*}$ can be achieved if and only if

$$
\begin{aligned}
& \max _{\phi_{m}} A\left|\boldsymbol{G}^{H} \boldsymbol{\Phi} \boldsymbol{H}+g^{s s}\right|^{2}-\lambda^{*}\left|g^{s m}+\boldsymbol{F}^{H} \boldsymbol{\Phi} \boldsymbol{H}\right|^{2} \\
& =A\left|\boldsymbol{G}^{H} \boldsymbol{\Phi}^{*} \boldsymbol{H}+g^{s s}\right|^{2}-\lambda^{*}\left|g^{s m}+\boldsymbol{F}^{H} \boldsymbol{\Phi}^{*} \boldsymbol{H}\right|^{2} \\
& =0 .
\end{aligned}
$$

where $\left\{\phi_{m}\right\}$ is any feasible solution of $\mathbf{P 2}$ to satisfy the constraint $C_{2}, \mathbf{\Phi}^{*}$ is the optimal phase shift reflection coefficients.

\section{Proof: See Appendix A.}

Theorem 1 shows that for an optimization problem with a fractional objective function, there exists an equivalent function in subtractive form, e.g., $A\left|\boldsymbol{G}^{H} \boldsymbol{\Phi} \boldsymbol{H}+g^{s s}\right|^{2}-$ $\lambda^{*}\left|g^{s m}+\boldsymbol{F}^{H} \boldsymbol{\Phi} \boldsymbol{H}\right|^{2}$ in the considered case. As a result, we focus on the equivalent objective function in the rest of the paper.

Based on the Dinkelbach's method [29], we obtain the following equivalent optimization problem

$$
\begin{gathered}
\mathbf{P 3} \max _{\phi_{m}} A\left|\boldsymbol{G}^{H} \boldsymbol{\Phi} \boldsymbol{H}+g^{s s}\right|^{2}-\lambda\left|g^{s m}+\boldsymbol{F}^{H} \boldsymbol{\Phi} \boldsymbol{H}\right|^{2} \\
\text { s.t. } \bar{C}_{2}:\left|\phi_{m}\right|=1, \forall m .
\end{gathered}
$$

The objective function in $\mathbf{P 3}$ is a strictly continuous and decreasing function with respect to the variable $\lambda$.

Theorem 2: For any $\Phi$ and $\lambda, Q(\lambda)=$ $\max _{\phi_{m}} A\left|\boldsymbol{G}^{H} \boldsymbol{\Phi} \boldsymbol{H}+g^{s s}\right|^{2}-\lambda\left|g^{s m}+\boldsymbol{F}^{H} \boldsymbol{\Phi} \boldsymbol{H}\right|^{2}$ is a strictly monotonic decreasing function with respect to the variable $\lambda$, and $Q(\lambda) \geq 0$.

Proof: See Appendix B.

Since $\boldsymbol{\phi}=\left[\phi_{1}, \phi_{2}, \cdots, \phi_{M}\right]^{H}, \overline{\boldsymbol{G}}=\operatorname{diag}(\boldsymbol{G}) \boldsymbol{H}$ and $\overline{\boldsymbol{F}}=$ 
$\operatorname{diag}(\boldsymbol{F}) \boldsymbol{H} . \mathbf{P 3}$ becomes

$$
\begin{aligned}
\overline{\boldsymbol{P}} 3 \max _{\phi_{m}} \boldsymbol{\phi}^{H}\left(A \overline{\boldsymbol{G}} \overline{\boldsymbol{G}}^{H}-\lambda \overline{\boldsymbol{F}} \overline{\boldsymbol{F}}^{H}\right) \boldsymbol{\phi}+A\left|g^{s s}\right|^{2}-\lambda\left|g^{s m}\right|^{2} \\
+\operatorname{Re}\left\{\left(A g^{s s} \overline{\boldsymbol{G}}^{H}-\lambda g^{s m} \overline{\boldsymbol{F}}^{H}\right) \boldsymbol{\phi}\right\} \\
+\operatorname{Re}\left\{\boldsymbol{\phi}^{H}\left(A g^{s s} \overline{\boldsymbol{G}}-\lambda g^{s m} \overline{\boldsymbol{F}}\right)\right\} \\
\text { s.t. } \bar{C}_{2}:\left|\phi_{m}\right|=1 .
\end{aligned}
$$

Define $\tilde{\boldsymbol{G}}=A \overline{\boldsymbol{G}} \overline{\boldsymbol{G}}^{H}-\lambda \overline{\boldsymbol{F}} \overline{\boldsymbol{F}}^{H} \in \mathcal{C}^{M \times M}, \overline{\boldsymbol{H}}=A g^{s s} \overline{\boldsymbol{G}}-$ $\lambda g^{s m} \overline{\boldsymbol{F}} \in \mathcal{C}^{M}$, and $\bar{A}=A\left|g^{s s}\right|^{2}-\lambda\left|g^{s m}\right|^{2}$, then $\overline{\boldsymbol{P}} 3$ becomes

$$
\mathbf{P 4} \max _{\phi_{m}} \boldsymbol{\phi}^{H} \tilde{\boldsymbol{G}} \boldsymbol{\phi}+\operatorname{Re}\left\{\boldsymbol{\phi}^{H} \overline{\boldsymbol{H}}\right\}+\operatorname{Re}\left\{\overline{\boldsymbol{H}}^{H} \boldsymbol{\phi}\right\}+\bar{A}
$$$$
\text { s.t. } \bar{C}_{2}:\left|\phi_{m}\right|=1 \text {. }
$$

Note that $\mathbf{P 4}$ is a non-convex quadratically constrained quadratic program (QCQP) problem, which can be converted into a homogeneous QCQP problem.

Specifically, by introducing an auxiliary variable $t, \mathbf{P 4}$ can be equivalently rewritten as

$$
\begin{array}{r}
\overline{\mathbf{P} 4} \max _{\bar{\phi}_{m}} \bar{\phi}^{H} \boldsymbol{M} \overline{\boldsymbol{\phi}}+\bar{A} \\
\text { s.t. } \tilde{C}_{2}:\left|\bar{\phi}_{m}\right|=1,
\end{array}
$$

where

$$
\boldsymbol{M}=\left[\begin{array}{cc}
\tilde{\boldsymbol{G}} & \overline{\boldsymbol{H}} \\
\overline{\boldsymbol{H}}^{H} & 0
\end{array}\right] \text { and } \overline{\boldsymbol{\phi}}=\left[\begin{array}{l}
\phi \\
t
\end{array}\right] .
$$

However, $\overline{\mathbf{P}} \mathbf{4}$ is still non-convex. Note that $\bar{\phi}^{H} \boldsymbol{M} \bar{\phi}=$ $\operatorname{Trace}\left(\boldsymbol{M} \bar{\phi} \bar{\phi}^{H}\right)$. Define $\boldsymbol{X}=\bar{\phi} \bar{\phi}^{H}$, which satisfies $\boldsymbol{X} \succcurlyeq 0$ and $\operatorname{rank}(\boldsymbol{X})=1$. As a result, problem $\overline{\mathbf{P}} \mathbf{4}$ becomes

$$
\begin{gathered}
\text { P5 } \max _{\boldsymbol{X}} \operatorname{Trace}(\boldsymbol{M} \boldsymbol{X})+\bar{A} \\
\text { s.t. } \bar{C}_{3}: \boldsymbol{X}_{m}=1, \forall m, \\
C_{4}: \boldsymbol{X} \succcurlyeq 0 .
\end{gathered}
$$

P5 is a convex SDP problem, it can be efficiently solved by using the convex optimization tool, such as SeDuMi [33] and YALMIP [34].

Generally, the solution $\boldsymbol{X}$ of $\mathbf{P 5}$ may not satisfy its rank constraint, namely, $\operatorname{rank}(\boldsymbol{X}) \neq 1$, therefore the optimal objective value of P5 only serves an upper bound of $\mathbf{P 4}$. To solve this problem and obtain a rank-one solution, the Gaussian randomization scheme and the singular-valuedecomposition scheme are used [17], [21], [35]. The iterative RA algorithm is summarized in Algorithm 1.

\section{Multi-user Resource Allocation Algorithm}

In this section, we extend the system model to the communication scenario where there are multiple users in each small cell and multiple MCUs in the macrocell. It is assumed that there are $K$ SCUs and $N$ MCUs, denoted the set as $\mathcal{K}=$ $\{1,2, \cdots, K\}, \forall k \in \mathcal{K}$ and $\mathcal{N}=\{1,2, \cdots, N\}, \forall n \in \mathcal{N}$. The superposition signals from the SBS can be expressed as

$$
x=\sum_{k=1}^{K} \sqrt{p_{k}} x_{k},
$$

where $p_{k}$ and $x_{k}$ denote the allocated power and signal from the SBS to the $k$-th SCU, respectively. Therefore, the signal

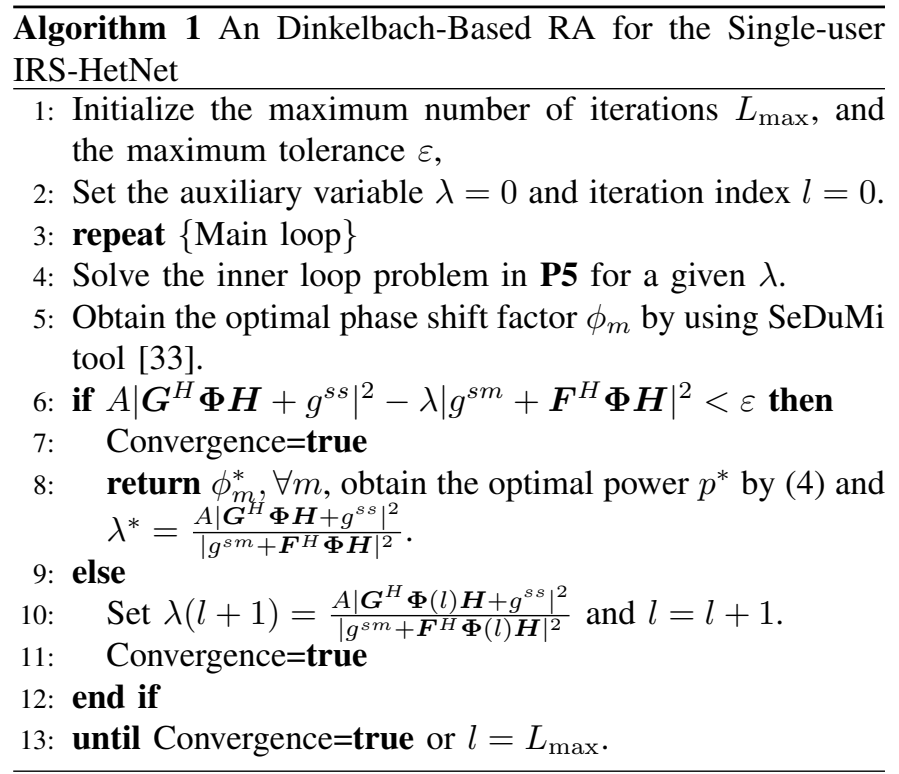

received at the $k$-th SCU is

$$
y_{k}=g_{k}^{s s} x+\boldsymbol{G}_{k}^{H} \boldsymbol{\Phi} \boldsymbol{H} x+n_{k},
$$

where $g_{k}^{s s}$ denotes the channel response from the SBS to the $k$-th SCU, $\boldsymbol{G}_{k}$ denotes the channel response from the IRS to the $k$-th SCU, $n_{k}=\sum_{n=1}^{N} P_{n} h_{k}^{m s}+\sigma_{k}$ denotes the sum of interference plus noise at the $k$-th SCU, where the background noise $\sigma_{k}$ satisfies $\sigma_{k} \sim \mathcal{C N}\left(0, \sigma^{2}\right) . h_{k}^{m s}$ is the channel response from the MBS to the $k$-th SCU, $P_{n}$ is the allocated power from the MBS to the $n$-th MCU. Since the MBS is often far from the indoor SCUs, the interference power $\sum_{n}^{N} P_{n} h_{k}^{m s}$ can be omitted [36]. Thus, the SINR at the $k$-th SCU can be expressed as

$$
\gamma_{k}^{\mathrm{SCU}}=\frac{p_{k}\left|g_{k}^{s s}+\boldsymbol{G}_{k}^{H} \mathbf{\Phi} \boldsymbol{H}\right|^{2}}{\left|g_{k}^{s s}+\boldsymbol{G}_{k}^{H} \mathbf{\Phi} \boldsymbol{H}\right|^{2} \sum_{i \neq k}^{K} p_{i}+\sigma^{2}} .
$$

Therefore, the achieved total rate for all SCUs is

$$
R=\sum_{k=1}^{K} \log _{2}\left(1+\gamma_{k}^{\mathrm{SCU}}\right) \text {. }
$$

The SINR received at the $n$-th MCU is

$$
\gamma_{n}^{\mathrm{MCU}}=\frac{P_{n}\left|h_{n}^{m m}\right|^{2}}{\sum_{k=1}^{K} p_{k}\left|g_{n}^{s m}+\boldsymbol{F}_{n}^{H} \boldsymbol{\Phi} \boldsymbol{H}\right|^{2}+\sigma^{2}},
$$

where $h_{n}^{m m}$ denotes the channel response from the MBS to the $n$-th MCU, and $g_{n}^{s m}$ is the channel gain from the SBS to the $n$-th MCU.

In order to improve the spectral efficiency, the sum rate achieved by all SCUs can be maximized by solving the 
following problem

P6

$$
\begin{aligned}
& \max _{p_{k}, \phi_{m}} \sum_{k=1}^{K} \log _{2}\left(1+\gamma_{k}^{\mathrm{SCU}}\right) \\
& \text { s.t. } \quad \bar{C}_{2}:\left|\phi_{m}\right|=1, \forall m, \\
& \quad C_{5}: \sum_{k=1}^{K} p_{k} \leq p^{\max }, \\
& C_{6}: \gamma_{n}^{\mathrm{MCU}} \geq \gamma_{n}^{\min }, \forall n,
\end{aligned}
$$

where $\gamma_{n}^{\min }$ denotes the minimum SINR required by the $n$-th MCU. However, it is difficult to obtain the optimal solution to P6, since the objective function is non-concave with respect to either $p_{k}$ or $\phi_{m}$. The constraint $C_{6}$ is non-convex due to the coupled variables $p_{k}$ and $\phi_{m}$. As the multiple variables are coupled in P6, it it difficult to obtain the globally optimal solutions. Thus, we propose a low-complexity algorithm for solving P6 approximately in the following section.

Since the variables $p_{k}$ and $\phi_{m}$ are coupled in $C_{6}$ and the objective function, we develop an efficient algorithm for P6 which optimizes $p_{k}$ and $\phi_{m}$ by using the AOM.

1) Optimizing $p_{k}$ with given $\phi_{m}$ : Based on $C_{6}$, we have

$$
\begin{aligned}
I_{n}^{t h} & =\frac{P_{n}\left|h_{n}^{m m}\right|^{2}}{\gamma_{n}^{\text {min }}}-\sigma^{2} \\
& \geq \sum_{k=1}^{K} p_{k}\left|g_{n}^{s m}+\boldsymbol{F}_{n}^{H} \mathbf{\Phi} \boldsymbol{H}\right|^{2},
\end{aligned}
$$

where $I_{n}^{t h}$ denotes the equivalent interference threshold [30], [31]. As a result, the power allocation problem becomes

$$
\begin{aligned}
& \mathbf{P 7} \quad \max _{p_{k}} \sum_{k=1}^{K} \log _{2}\left(1+\gamma_{k}^{\mathrm{SCU}}\right) \\
& \text { s.t. } C_{5}: \sum_{k=1}^{K} p_{k} \leq p^{\max }, \\
& \bar{C}_{6}: \sum_{k=1}^{K} p_{k}\left|g_{n}^{s m}+\boldsymbol{F}_{n}^{H} \mathbf{\Phi} \boldsymbol{H}\right|^{2} \leq I_{n}^{t h}, \forall n .
\end{aligned}
$$

It is noted that $C_{5}$ and $\bar{C}_{6}$ are linear constraints. But the objective function is nonconvex. Based on the SCALE algorithm in [32], for any $z>0$, we have the following bound

$$
\log _{2}(1+z) \geq \alpha \log _{2} z+\beta
$$

where $\alpha=\frac{z_{0}}{1+z_{0}}$ and $\beta=\log _{2}\left(1+z_{0}\right)-\alpha \log _{2} z_{0}$ are the auxiliary variables. The relationship is tight at $z=z_{0}$, and $\alpha$, $\beta$ are the approximation constants. Therefore, we define

$$
R=\sum_{k=1}^{K} \alpha_{k} \log _{2} \gamma_{k}+\sum_{k=1}^{K} \beta_{k},
$$

which is a lower bound of the sum rate achieved by all SCUs. Note that the relaxation is still a nonconvex form due to the d.c. (difference of convex) structure. With the Logarithmic transformation $\bar{p}_{k}=\ln p_{k}, \forall k$, we have the following convex optimization problem

$$
\begin{gathered}
\mathbf{P 8} \max _{\bar{p}_{k}}-\sum_{k=1}^{K} \alpha_{k} \log _{2}\left(\sigma^{2}+\left|g_{k}^{s s}+\boldsymbol{G}_{k}^{H} \mathbf{\Phi} H\right|^{2} \sum_{i \neq k}^{K} e^{\bar{p}_{i}}\right) \\
+\sum_{k=1}^{K} \alpha_{k} \log _{2}\left(\left|g_{k}^{s s}+\boldsymbol{G}_{k}^{H} \mathbf{\Phi} H\right|^{2}\right) \\
+\sum_{k=1}^{K} \alpha_{k} \bar{p}_{k}-\sum_{k=1}^{K} \beta_{k} \\
\text { s.t. } \bar{C}_{5}: \sum_{k=1}^{K} e^{\bar{p}_{k}} \leq p^{\max }, \\
\tilde{C}_{6}: \sum_{k=1}^{K} e^{\bar{p}_{k}}\left|g_{n}^{s m}+\boldsymbol{F}_{n}^{H} \mathbf{\Phi} H\right|^{2} \leq I_{n}^{t h}, \forall n .
\end{gathered}
$$

Note that $\mathbf{P 8}$ is a standard concave maximization problem since the log-sum-exp is convex [37]. Considering the convexity of P8, the optimal power allocation can be obtained by the Lagrange dual function as

$$
\begin{aligned}
& L\left(\bar{p}_{k}, \mu, \vartheta_{n}\right)=\sum_{k=1}^{K} \alpha_{k} \bar{p}_{k}-\sum_{k=1}^{K} \beta_{k}+\mu\left(p^{\max }-\sum_{k=1}^{K} e^{\bar{p}_{k}}\right) \\
& \quad-\sum_{k=1}^{K} \alpha_{k} \log _{2}\left(\sigma^{2}+\left|g_{k}^{s s}+\boldsymbol{G}_{k}^{H} \mathbf{\Phi} \boldsymbol{H}\right|^{2} \sum_{i \neq k}^{K} e^{\bar{p}_{i}}\right) \\
& \quad+\sum_{n=1}^{N} \vartheta_{n}\left(I_{n}^{t h}-\sum_{k=1}^{K} e^{\bar{p}_{k}}\left|g_{n}^{s m}+\boldsymbol{F}_{n}^{H} \mathbf{\Phi} \boldsymbol{H}\right|^{2}\right) \\
& \quad+\sum_{k=1}^{K} \alpha_{k} \log _{2}\left(\left|g_{k}^{s s}+\boldsymbol{G}_{k}^{H} \mathbf{\Phi} \boldsymbol{H}\right|^{2}\right)
\end{aligned}
$$

where $\mu$ and $\vartheta_{n}$ are the non-negative Lagrange multipliers. Based on the same approach, we have the following closedform solution

$$
p_{k}=\left[\frac{\alpha_{k}}{\mu+\sum_{n=1}^{N} \vartheta_{n}\left|g_{n}^{s m}+\boldsymbol{F}_{n}^{H} \boldsymbol{\Phi} \boldsymbol{H}\right|^{2}}\right]^{+},
$$

where $[x]^{+}=\max \{0, x\}$.

Based on the sub-gradient method, the Lagrange multipliers can be calculated by

$$
\begin{gathered}
\mu^{l+1}=\left[\mu^{l}-d_{1}^{l} \times\left(p^{\max }-\sum_{k=1}^{K} p_{k}\right)\right]^{+} \\
\vartheta_{n}^{l+1}=\left[\vartheta_{n}^{l}-d_{2}^{l} \times\left(I_{n}^{t h}-\sum_{k=1}^{K} p_{k}\left|g_{n}^{s m}+\boldsymbol{F}_{n}^{H} \mathbf{\Phi} \boldsymbol{H}\right|^{2}\right)\right]^{+},
\end{gathered}
$$

where $l$ is the iteration number. $d_{1}^{l}$ and $d_{2}^{l}$ denote the sufficiently small positive step sizes satisfying $\sum_{l=1}^{\infty} d_{i}^{l}=$ $\infty, \lim _{l \rightarrow \infty} d_{i}^{l}=0, \forall i=\{1,2\}$ [31]. The dual variables $\mu(l)$ and $\vartheta_{n}^{\infty}(l)$ will converge to the optimal values $\mu^{*}$ and $\vartheta_{n}^{*}, \forall n$ as $l \rightarrow \infty$ [38], [39]. Note that the duality gap for the problem 
P8 is zero and the solution is unique. As a result, the primal variable $p_{k}^{*}\left(\mu(l), \vartheta_{n}(l)\right)$ can reach its optimal value $p_{k}^{*}, \forall k$.

2) Optimizing $\phi_{m}$ with given $p_{k}$ : Now we perform the optimization over $\phi_{m}$ with the fixed $p_{k}$. Then $\mathbf{P 8}$ is equivalent to

P9

$$
\begin{aligned}
\max _{\phi_{m}} & \sum_{k=1}^{K} \frac{\alpha_{k} p_{k}\left|g_{k}^{s s}+\boldsymbol{G}_{k}^{H} \boldsymbol{\Phi} \boldsymbol{H}\right|^{2}}{\left|g_{k}^{s s}+\boldsymbol{G}_{k}^{H} \mathbf{\Phi} \boldsymbol{H}\right|^{2} \sum_{i \neq k}^{K} p_{i}+\sigma^{2}} \\
\text { s.t. } & \bar{C}_{2}:\left|\phi_{m}\right|=1, \forall m, \\
& \bar{C}_{6}: \sum_{k=1}^{K} p_{k}\left|g_{n}^{s m}+\boldsymbol{F}_{n}^{H} \mathbf{\Phi} \boldsymbol{H}\right|^{2} \leq I_{n}^{t h}, \forall n,
\end{aligned}
$$

P9 is the sum of multiple-ratio fractional programming problems, and the non-convexity introduced by the fractional objective function and the non-convex unit-modulus constraint $C_{4}$. We adopt a relaxation method to solve $\mathbf{P 9}$ efficiently.

Define $\tilde{\boldsymbol{F}}=\overline{\boldsymbol{F}} \overline{\boldsymbol{F}}^{H}$, based on $|x+y| \leq|x|+|y|$, and $\overline{\boldsymbol{F}}=\operatorname{diag}\left(\boldsymbol{F}_{n}\right) \boldsymbol{H}$, the constraint $\bar{C}_{5}$ becomes

$$
\begin{aligned}
\left|g_{n}^{s m}+\boldsymbol{F}_{n}^{H} \mathbf{\Phi} \boldsymbol{H}\right|^{2} & \leq\left|g_{n}^{s m}\right|^{2}+\left|\boldsymbol{\phi}^{H} \overline{\boldsymbol{F}}\right|^{2} \\
& =\left|g_{n}^{s m}\right|^{2}+\boldsymbol{\phi}^{H} \tilde{\boldsymbol{F}} \boldsymbol{\phi} \leq \frac{I_{n}^{t h}}{\sum_{k=1}^{K} p_{k}} .
\end{aligned}
$$

Thus we have

$$
\phi^{H} \tilde{\boldsymbol{F}} \boldsymbol{\phi} \leq \bar{I}_{n}^{t h}, \forall n,
$$

where $\bar{I}_{n}^{t h}=\frac{I_{n}^{t h}}{\sum_{k=1}^{K} p_{k}}-\left|g_{n}^{s m}\right|^{2}$ is the equivalent interference level for the $n$-th MCU. Thus, $\mathbf{P 9}$ can be reformulated as

$$
\begin{aligned}
\mathbf{P 1 0} \max _{\phi_{m}, y_{k}} & \sum_{k=1}^{K} y_{k} \\
\text { s.t. } \bar{C}_{2}:\left|\phi_{m}\right|=1, \forall m, & \\
C_{7} & : \frac{\alpha_{k} p_{k}\left|g_{k}^{s s}+\boldsymbol{G}_{k}^{H} \boldsymbol{\Phi} \boldsymbol{H}\right|^{2}}{\left|g_{k}^{s s}+\boldsymbol{G}_{k}^{H} \boldsymbol{\Phi} \boldsymbol{H}\right|^{2} \sum_{i \neq k}^{K} p_{i}+\sigma^{2}} \geq y_{k}, \forall k, \\
C_{8} & : \boldsymbol{\phi}^{H} \tilde{\boldsymbol{F}} \boldsymbol{\phi} \leq \bar{I}_{n}^{t h}, \forall n,
\end{aligned}
$$

where $y_{k} \geq 0$ is an auxiliary variable. Define $\overline{\boldsymbol{G}}_{k}=$ $\operatorname{diag}\left(\boldsymbol{G}_{k}\right) \boldsymbol{H}, C_{7}$ can be rewritten as

$$
\boldsymbol{\phi}^{H} \overline{\boldsymbol{G}}_{k} \overline{\boldsymbol{G}}_{k}^{H} \boldsymbol{\phi}+2 \operatorname{Re}\left\{g_{k}^{s s, *} \overline{\boldsymbol{G}}_{k}^{H} \boldsymbol{\phi}\right\}+f_{k} \leq 0
$$

where $(\cdot)^{*}$ denotes the conjugate of vector, $\operatorname{Re}(\cdot)$ denotes the real part, and $f_{k}=\left|g_{k}^{s s}\right|^{2}+\frac{y_{k} \sigma^{2}}{y_{k} \sum_{i \neq k}^{K} p_{i}-\alpha_{k} p_{k}}$. What's more, $\bar{C}_{2}$ can be rewritten as

$$
\boldsymbol{\phi}^{H} \boldsymbol{U} \boldsymbol{\phi}=1 \text {, }
$$

where $\boldsymbol{U} \in R^{M \times M}$ is a symmetric matrix with elements of zeros, except for $u_{m, m}=1$.
Thus, we have

$$
\mathbf{P 1 1} \begin{aligned}
& \max _{\boldsymbol{\phi}, y_{k}} \sum_{k=1}^{K} y_{k} \\
& \text { s.t. } C_{4}: \boldsymbol{\phi}^{H} \boldsymbol{U} \boldsymbol{\phi}=1, \\
& \bar{C}_{5}: \boldsymbol{\phi}^{H} \tilde{\boldsymbol{F}} \boldsymbol{\phi} \leq \bar{I}_{n}^{t h}, \forall n, \\
& C_{6}: \bar{f}_{k}+f_{k} \leq 0,
\end{aligned}
$$

where $\bar{f}_{k}=\boldsymbol{\phi}^{H} \overline{\boldsymbol{G}}_{k} \overline{\boldsymbol{G}}_{k}^{H} \boldsymbol{\phi}+2 \operatorname{Re}\left\{g_{k}^{s s, *} \overline{\boldsymbol{G}}_{k}^{H} \boldsymbol{\phi}\right\}$. P11 is a convex optimization problem with the quadratic constraints. Thus, the Lagrangian of $\mathbf{P 1 1}$ is

$$
\begin{aligned}
& L\left(\phi, y_{k}, \kappa, \rho_{n}, \varpi_{k}\right)=-\sum_{k=1}^{K} y_{k}+\kappa\left(\phi^{H} \boldsymbol{U} \boldsymbol{\phi}-1\right) \\
& +\sum_{k=1}^{K} \varpi_{k}\left(\bar{f}_{k}+f_{k}\right)+\sum_{n=1}^{N} \rho_{n}\left(\phi^{H} \tilde{\boldsymbol{F}} \boldsymbol{\phi}-\bar{I}_{n}^{t h}\right),
\end{aligned}
$$

where $\kappa, \varpi_{k}, \rho_{n}$ are the Lagrange multipliers. Based on KKT conditions [37], the optimal value of $y_{k}$ can be obtained as

$$
y_{k}^{*}=\left[\frac{\alpha_{k} p_{k}+\sigma \sqrt{\varpi_{k} \alpha_{k} p_{k}}}{\sum_{j \neq k}^{K} p_{i}}\right]^{+},
$$

where the Lagrange multipliers can be updated by using the subgradient methods, i.e.,

$$
\begin{gathered}
\kappa^{l+1}=\left[\kappa^{l}+d_{3}^{l} \times\left(\phi^{H} \boldsymbol{U} \boldsymbol{\phi}-1\right)\right]^{+}, \\
\rho_{n}^{l+1}=\left[\rho_{n}^{l}+d_{4}^{l} \times\left(\boldsymbol{\phi}^{H} \tilde{\boldsymbol{F}} \boldsymbol{\phi}-\bar{I}_{n}^{t h}\right)\right]^{+}, \\
\varpi_{k}^{l+1}=\left[\varpi_{k}^{l}+d_{5}^{l} \times\left(\bar{f}_{k}+f_{k}\right)\right]^{+},
\end{gathered}
$$

where $d_{3}^{l}, d_{4}^{l}$ and $d_{5}^{l}$ are the step sizes.

In order to solve the phase shift of the IRS, based on a Schur complement, the dual problem of $\mathbf{P 1 1}$ can be formulated as

P12

$$
\begin{gathered}
\max \gamma \\
\text { s.t. } \gamma \geq 0, \\
\tilde{\boldsymbol{M}} \succcurlyeq 0,
\end{gathered}
$$

where $\tilde{\boldsymbol{M}}=\left[\begin{array}{cc}B_{1} & B_{2} \\ B_{2}^{T} & B_{3}\end{array}\right], \quad B_{1}=$ $\kappa \boldsymbol{U}+\sum_{k=1}^{K} \varpi_{k} \overline{\boldsymbol{G}} \overline{\boldsymbol{G}}^{H}+\sum_{n=1}^{N} \rho_{n} \tilde{\boldsymbol{F}}, B_{2}=\sum_{k=1}^{K} \varpi_{k} R e\left\{g_{k}^{s s, *} \overline{\boldsymbol{G}}_{k}^{H}\right\}$, and $B_{3}=\sum_{k=1}^{K} \varpi_{k} f_{k}-\sum_{k=1}^{K} y_{k}-\kappa-\sum_{n=1}^{N} \rho_{n} \bar{I}_{n}^{t h}-\gamma \cdot \gamma$ is an auxiliary variable. P12 is an SDP problem and the Slater's constraint qualification is satisfied [37]. Particularly, an iterative-based RA algorithm for the multiuser IRS-based HetNet is summarized in Algorithm 2.

\section{Simulation Results}

In this section, simulation results are given to investigate the performance of the proposed RA scheme in the IRS-enabled HetNet. A spectrum-sharing small cell is randomly distributed in the coverage area of the macrocell. SCUs are uniformly distributed in the coverage area of their associated SBS. The 


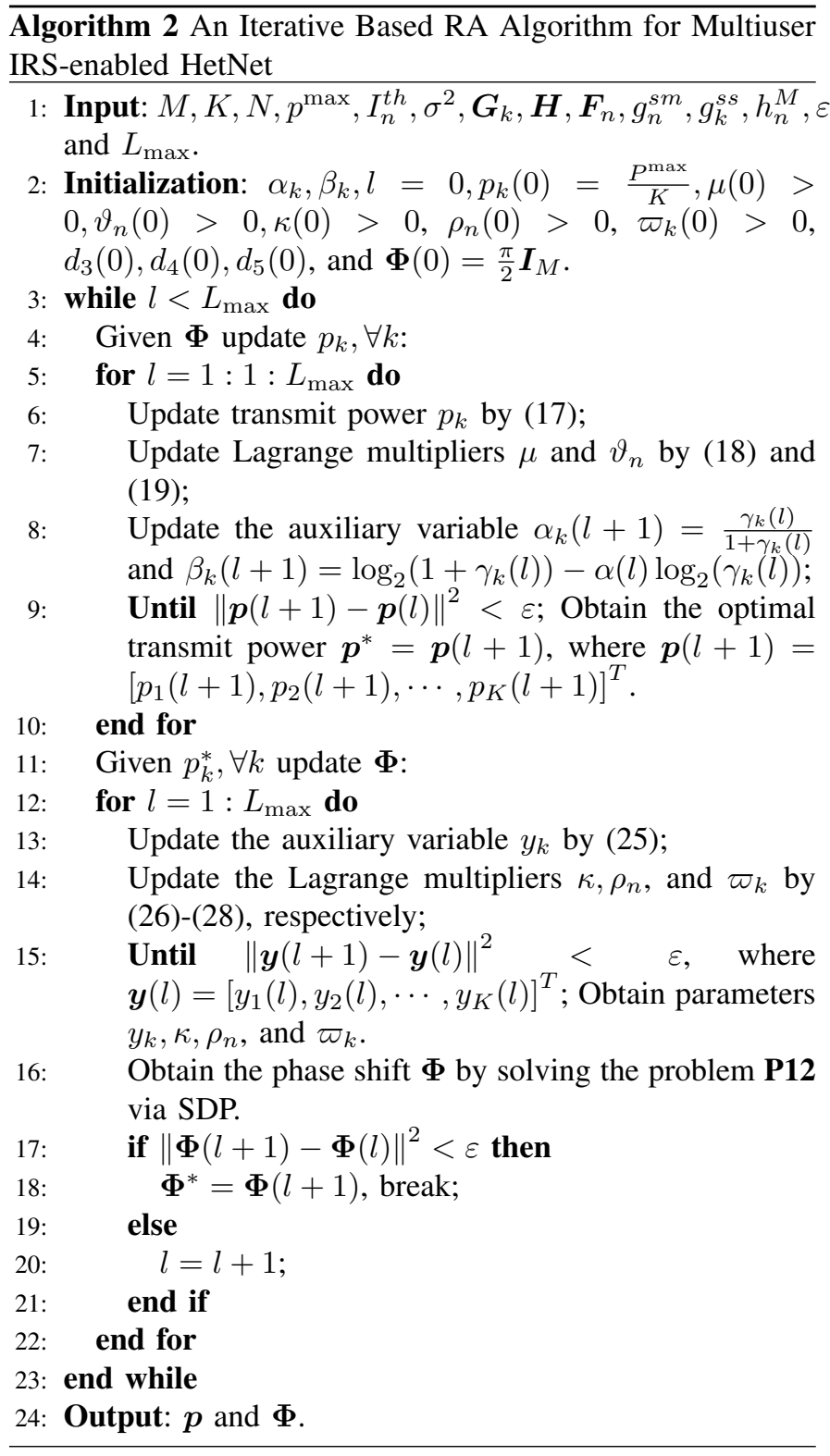

coverage radii of the macrocell and small cell are $500 \mathrm{~m}$ and 20 $\mathrm{m}$, respectively. The distance between the SBS and the IRS is $10 \mathrm{~m}$. The channel model is the same as in [31]. The stopping criterion for convergence is $\varepsilon=10^{-6}$. The noise power is $\sigma^{2}=-100 \mathrm{dBm}$. The channel bandwidth is $10 \mathrm{MHz}$ [40]. For performance comparison, we provide the traditional RA scheme without IRS (e.g., $\mathbf{\Phi}=0$ ) as the benchmark.

\section{A. Single-user Case}

First, we consider a single-user IRS-enabled HetNet with the target SINR of MCU $\gamma^{\text {min }}=2 \mathrm{~dB}$. The unit bandwidth is considered for simplicity. Fig. 2 depicts the data rate of the SCU versus the transmit power at the MBS. From the figure, the data rate of the SCU improves quickly as the transmit power of the MBS increases. The reason is that, the large transmit power of the MBS (e.g., $P$ ) increases the feasible region of transmit power of the SCU from the constraint $C 1$, which allows more transmit power from the SBS to improve

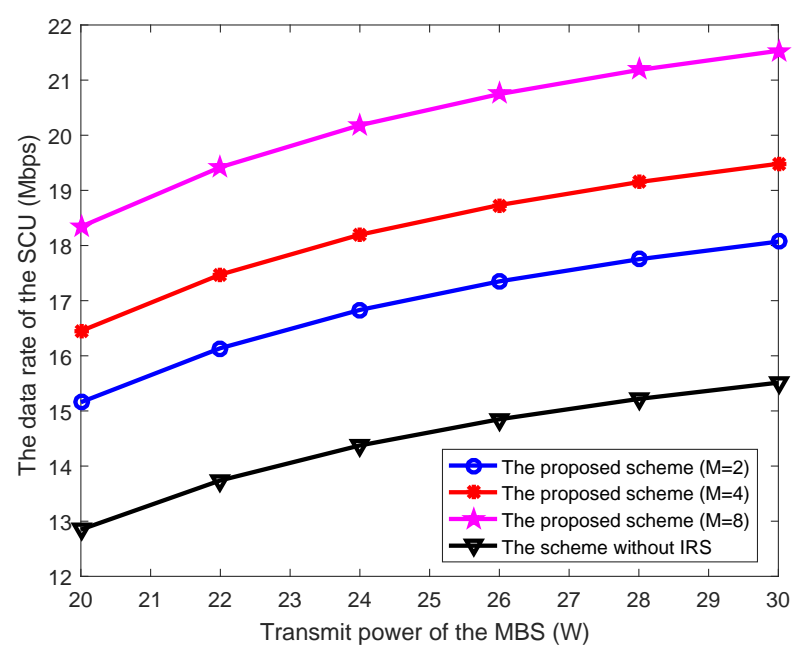

Fig. 2. The achieved data rate of the SCU under different transmit power of MBS.

system performance. Besides, the figure also shows that the data rate under the proposed scheme is better than that of the scheme without IRS, which indicates that the IRS can further improve the performance of HetNets in a passive reflecting way. Moreover, with the increasing number of reflecting elements at the IRS, the data rate increases heavily, which indicates that a larger number of passive reflecting elements leads to better performance.

Fig. 3 shows the data rate of the SCU versus the minimum SINR requirement for the MCU, $\gamma^{\mathrm{min}}$. The transmit power of MBS is assumed to be $20 \mathrm{~W}$. With the increasing $\gamma^{\text {min }}$, the data rate of the SCU decreases considerably. Because under the fixed transmit power at the MBS, the allowed transmit power of SCU becomes smaller to guarantee less interference to the MCU for the SINR requirement. It is noted that in terms of the achieved transmission performance, i.e., the achieved data rate, the proposed scheme (e.g., data rate) is still better than that of the scheme without IRS. Moreover, the performance gap of the SCU under the two schemes becomes small since the small transmit power leads to less interference to the MCU.

Fig. 4 compares the transmit power at the SBS versus the number of reflecting elemets (e.g., $M$ ). First, it is observed that the required transmit power of the proposed scheme is significantly lower than that without the IRS under different transmit power at the MBS. This demonstrates that the energy consumption of the system can be reduced when the IRS is adopted. Moreover, one can observe that the received transmit power of the SBS decreases with the increasing number of reflecting elements. This is expected since the larger reflecting elements provide more multipath interference to the MCU. The available transmit power of the SBS degrades for guaranteeing the QoS of the MCU. Finally, when the transmit power at the MBS is increased, the available transmit power at the SBS is also increased. Because the large transmit power at the MBS can allow more transmit power of the SBS to improve the communication quality of the SCU according to (3). 


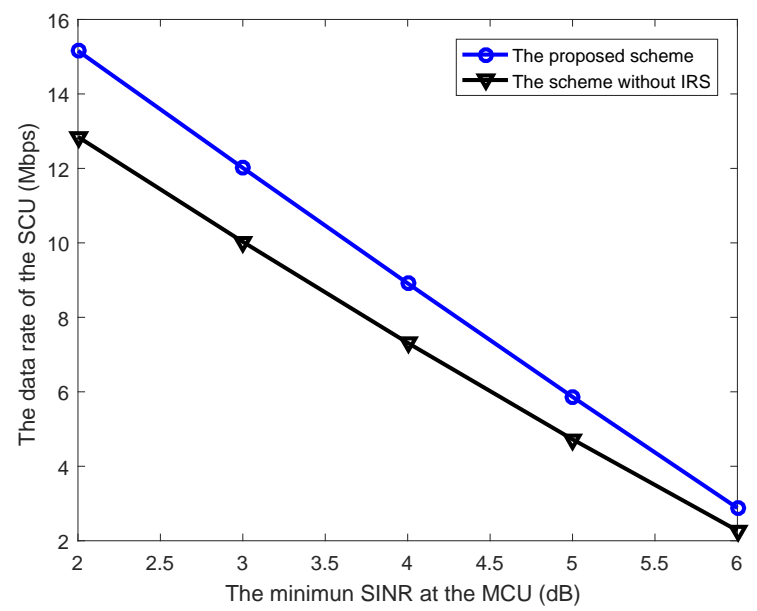

Fig. 3. The achieved data rate of the SCU under different SINR thresholds of the MCU.

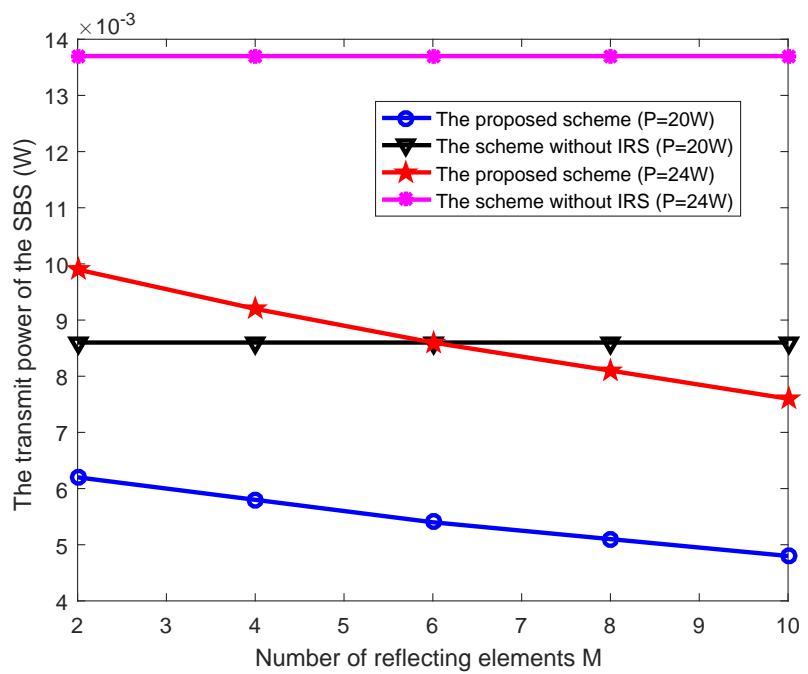

Fig. 4. Transmit power of SBS versus the number of reflecting elements.

\section{B. Multi-user Case}

Next, we consider a multiuser system with three SCUs $K=3$, two MCUs $N=2$ and four reflecting elements $M=4$. The SCUs are randomly distributed in the small cell network. Assume each MCU receiver has the same interference power threshold. The maximum transmit power of the SBS is $P^{\max }=1 \mathrm{~W}$, and the interference power threshold is $I_{n}^{t h}=10^{-3} \mathrm{~mW}$ [27], [31].

Before comparing the performance in the multi-user system, we first show the convergence behaviour of the proposed scheme in Fig. 5. It is observed that the transmit power allocated by the SBS to the SCUs can quickly reach the convergence within a small number of iterations (e.g., less than 8 iterations), which demonstrates good convergence of the proposed scheme in the multiuser IRS-enabled HetNet.

Fig. 6 shows the sum rate of all SCUs versus the number of reflecting elements with different SCUs. It is observed that the total data rate achieved by the SCUs increases with the larger reflecting element since it provides more reflecting signals to the SCUs. The reflecting signals can enhance signal strength

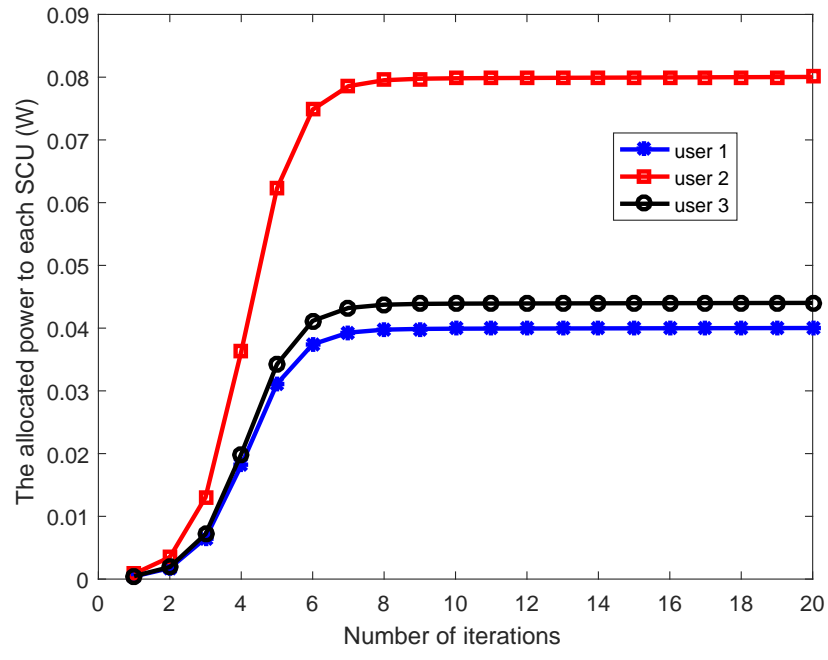

Fig. 5. Convergence behaviour of the proposed scheme.

at the receivers. However, the data rate achieved by all SCUs under the traditional scheme without IRS is not affected by the number of reflecting elements. Besides, the total rate of SCUs with larger number of users is bigger than that with small number of users. The rate gap under the small number of reflecting elements (e.g., $M=2$ ) is larger than that under the large number of reflecting elements (e.g., $M=10$ ). Because the interference among different users is small due to less transmission path, the total rate is decided by the number of users. Thus there is a big performance gap at the region with small reflecting elements. However, due to the effect of the cross-tier interference constraint (e.g., $\bar{C}_{5}$ ), the overall performance of the small cell is limited. It is impossible to unrestrictedly improve the performance with the increasing number of reflecting elements.

Fig. 7 shows the total rate of SCUs versus different interference thresholds of the MCU, $I_{n}^{t h}$. The total rate of SCUs increases with the increasing interference threshold of the MCU. The higher interference threshold means that the MCU can tolerate higher interference from other BSs and IRS reflection. Correspondingly, the SBS can allocate more transmit power to SCUs for obtaining higher data rate. Moreover, the proposed scheme with large number of reflecting elements achieves better performance than other two cases. The reason is that the IRS can enhance the system performance by providing multiple flexible transmission paths.

\section{CONCLUSIONS}

In this paper, we considered the downlink transmission in an IRS-enabled HetNet and presented two computationally efficient RA algorithms for optimizing the transmit power at the SBS and the phase shifts at the IRS under the single user and the multi-user scenarios, respectively. For the single-user case, we developed an optimal RA algorithm and obtained a closed-form solution. For the multi-user scenario, the RA algorithm was designed by using an alternating approach, where the non-convex problem is converted into an SDP problem by using the successive convex approximation. The 


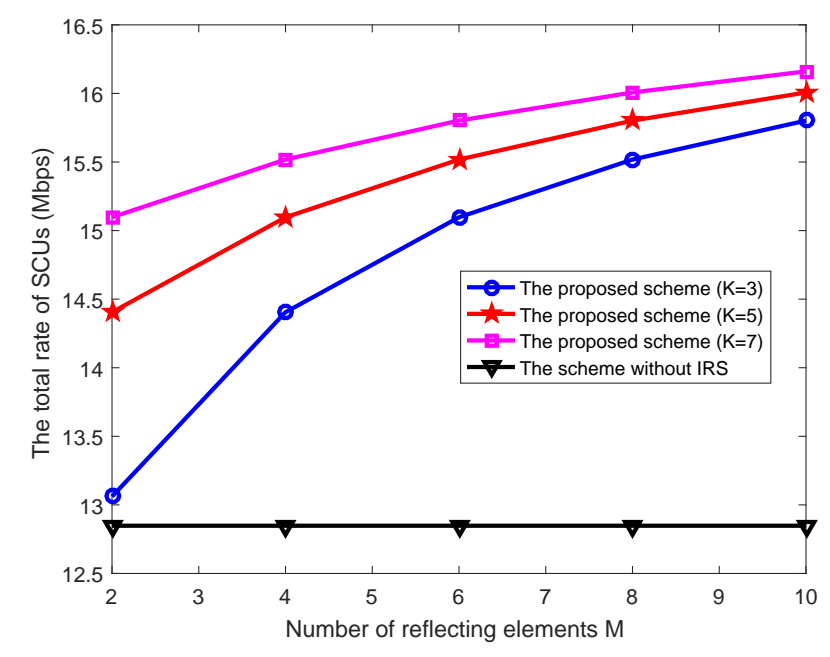

Fig. 6. The data rate of all SCUs under different number of reflecting elements.

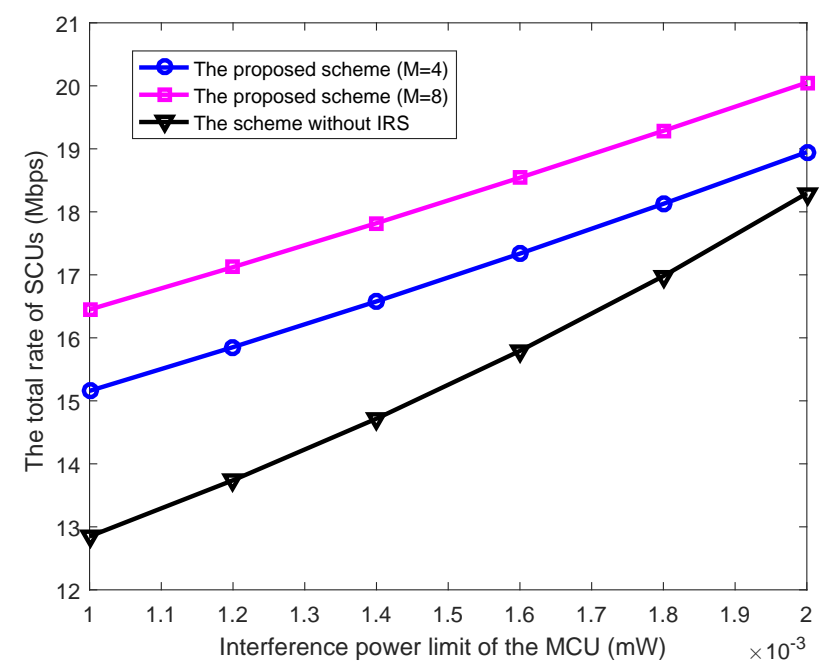

Fig. 7. The data rate of all SCUs under different interference power level of the MCU.

numerical results showed that the proposed algorithm can improve the total data rate of SCUs with guaranteeing the QoS of the MCU.

\section{APPENDIX A}

\section{ThE PROOF OF THEOREM 1}

By following a similar approach as in [29], the Theorem 1 can be proved by the following two steps.

First, the sufficient condition of Theorem 1 is proved. Define the maximum objective value as $\lambda^{*}=\frac{A\left|\boldsymbol{G}^{H} \boldsymbol{\Phi}^{*} \boldsymbol{H}+g^{s s}\right|^{2}}{\left|g^{s m}+\boldsymbol{F}^{H} \boldsymbol{\Phi}^{*} \boldsymbol{H}\right|^{2}}$, where $\boldsymbol{\Phi}^{*}=\left[\phi_{1}^{*}, \phi_{2}^{*}, \cdots, \phi_{M}^{*}\right]$ represents the optimal phase shift policy at the IRS. It is observed that $\lambda^{*}$ holds, i.e.,

$$
\lambda^{*}=\frac{A\left|\boldsymbol{G}^{H} \boldsymbol{\Phi}^{*} \boldsymbol{H}+g^{s s}\right|^{2}}{\left|g^{s m}+\boldsymbol{F}^{H} \boldsymbol{\Phi}^{*} \boldsymbol{H}\right|^{2}} \geq \frac{A\left|\boldsymbol{G}^{H} \boldsymbol{\Phi} \boldsymbol{H}+g^{s s}\right|^{2}}{\left|g^{s m}+\boldsymbol{F}^{H} \boldsymbol{\Phi} \boldsymbol{H}\right|^{2}},
$$

where $\boldsymbol{\Phi}$ is the feasible phase shift in P2. Then, based on
(A.1), we can obtain the following formulas:

$$
\left\{\begin{array}{l}
A\left|\boldsymbol{G}^{H} \boldsymbol{\Phi} \boldsymbol{H}+g^{s s}\right|^{2}-\lambda^{*}\left|g^{s m}+\boldsymbol{F}^{H} \boldsymbol{\Phi} \boldsymbol{H}\right|^{2} \leq 0 \\
A\left|\boldsymbol{G}^{H} \boldsymbol{\Phi}^{*} \boldsymbol{H}+g^{s s}\right|^{2}-\lambda^{*}\left|g^{s m}+\boldsymbol{F}^{H} \boldsymbol{\Phi}^{*} \boldsymbol{H}\right|^{2}=0 .
\end{array}\right.
$$

As a result, we have the conclusion that $\max _{\phi} A \mid \boldsymbol{G}^{H} \boldsymbol{\Phi} \boldsymbol{H}+$ $\left.g^{s s}\right|^{2}-\lambda^{*}\left|g^{s m}+\boldsymbol{F}^{H} \boldsymbol{\Phi} \boldsymbol{H}\right|^{2}=0$, and it is achievable under the optimal policy $\Phi^{*}$. The sufficient condition has been proved.

Second, the necessary condition is provided. Assume the optimal phase policy is $\overline{\boldsymbol{\Phi}}^{*}$, we have $A\left|\boldsymbol{G}^{H} \overline{\boldsymbol{\Phi}}^{*} \boldsymbol{H}+g^{s s}\right|^{2}$ $\lambda^{*}\left|g^{s m}+\boldsymbol{F}^{H} \overline{\boldsymbol{\Phi}}^{*} \boldsymbol{H}\right|^{2}=0$. For any feasible solution $\boldsymbol{\Phi}$, it can be written as

$$
\begin{aligned}
& A\left|\boldsymbol{G}^{H} \boldsymbol{\Phi} \boldsymbol{H}+g^{s s}\right|^{2}-\lambda^{*}\left|g^{s m}+\boldsymbol{F}^{H} \boldsymbol{\Phi} \boldsymbol{H}\right|^{2} \\
& \leq A\left|\boldsymbol{G}^{H} \overline{\boldsymbol{\Phi}}^{*} \boldsymbol{H}+g^{s s}\right|^{2}-\lambda^{*}\left|g^{s m}+\boldsymbol{F}^{H} \overline{\boldsymbol{\Phi}}^{*} \boldsymbol{H}\right|^{2} \\
& =0 .
\end{aligned}
$$

Based on (A.3), we have

$$
\left\{\begin{array}{l}
\frac{A\left|\boldsymbol{G}^{H} \boldsymbol{\Phi} \boldsymbol{H}+g^{s s}\right|^{2}}{\left|g^{s m}+\boldsymbol{F}^{H} \boldsymbol{\Phi} \boldsymbol{H}\right|^{2}} \leq \lambda^{*} \\
\frac{A\left|\boldsymbol{G}^{H} \overline{\boldsymbol{\Phi}}^{*} \boldsymbol{H}+g^{s s}\right|^{2}}{\left|g^{s m}+\boldsymbol{F}^{H} \overline{\boldsymbol{\Phi}}^{*} \boldsymbol{H}\right|^{2}}=\lambda^{*}
\end{array}\right.
$$

Therefore, the optimal phase shift policy $\overline{\boldsymbol{\Phi}}^{*}$ for the converted objective function is also the optimal RA scheme for the original objective function. Thus, the necessary condition of Theorem 1 is completed.

\section{APPENDIX B}

THE PRoOf OF THEOREM 2

According to $\mathbf{P 3}$, an equivalent objective function is given by

$$
Q(\lambda)=\max _{\phi_{m}} A\left|\boldsymbol{G}^{H} \boldsymbol{\Phi} \boldsymbol{H}+g^{s s}\right|^{2}-\lambda\left|g^{s m}+\boldsymbol{F}^{H} \boldsymbol{\Phi} \boldsymbol{H}\right|^{2} .
$$

Assume there are two optimal auxiliary variables $\lambda_{1}$ and $\lambda_{2}\left(\lambda_{1}>\lambda_{2}\right)$ for the optimal phase shift policies $\boldsymbol{\Phi}_{1}$ and $\boldsymbol{\Phi}_{2}$, respectively. Then

$$
\begin{aligned}
Q\left(\lambda_{2}\right) & =A\left|\boldsymbol{G}^{H} \boldsymbol{\Phi}_{\mathbf{2}} \boldsymbol{H}+g^{s s}\right|^{2}-\lambda_{2}\left|g^{s m}+\boldsymbol{F}^{H} \boldsymbol{\Phi}_{\mathbf{2}} \boldsymbol{H}\right|^{2} \\
& >A\left|\boldsymbol{G}^{H} \mathbf{\Phi}_{\mathbf{1}} \boldsymbol{H}+g^{s s}\right|^{2}-\lambda_{2}\left|g^{s m}+\boldsymbol{F}^{H} \mathbf{\Phi}_{\mathbf{1}} \boldsymbol{H}\right|^{2} \\
& >A\left|\boldsymbol{G}^{H} \boldsymbol{\Phi}_{\mathbf{1}} \boldsymbol{H}+g^{s s}\right|^{2}-\lambda_{1}\left|g^{s m}+\boldsymbol{F}^{H} \boldsymbol{\Phi}_{\mathbf{1}} \boldsymbol{H}\right|^{2} \\
& =Q\left(\lambda_{1}\right) .
\end{aligned}
$$

Therefore, $Q(\lambda)$ is a strictly decreasing function with the variable $\lambda$.

Define $\boldsymbol{\Phi}^{\prime}$ be any feasible solution for $\mathbf{P 3}$ and $\lambda^{\prime}=$ $\frac{A\left|\boldsymbol{G}^{H} \boldsymbol{\Phi}^{\prime} \boldsymbol{H}+g^{s s}\right|^{2}}{\left|g^{s m}+\boldsymbol{F}^{H} \boldsymbol{\Phi}^{\prime} \boldsymbol{H}\right|^{2}}$, we have

$$
\begin{aligned}
Q\left(\lambda^{\prime}\right) & =\max _{\phi_{m}} A\left|\boldsymbol{G}^{H} \boldsymbol{\Phi} \boldsymbol{H}+g^{s s}\right|^{2}-\lambda^{\prime}\left|g^{s m}+\boldsymbol{F}^{H} \boldsymbol{\Phi} \boldsymbol{H}\right|^{2} \\
& \geq A\left|\boldsymbol{G}^{H} \boldsymbol{\Phi}^{\prime} \boldsymbol{H}+g^{s s}\right|^{2}-\lambda^{\prime}\left|g^{s m}+\boldsymbol{F}^{H} \boldsymbol{\Phi}^{\prime} \boldsymbol{H}\right|^{2}=0 .
\end{aligned}
$$

As a result, we have $Q(\lambda) \geq 0$. The proof is completed.

\section{REFERENCES}

[1] C. Liaskos, S. Nie, A. Tsioliaridou, A. Pitsillides, S. Ioannidis and I. Akyildiz, "A new wireless communication paradigm through software- 
controlled metasurfaces," IEEE Commun. Mag., vol. 56, no. 9, pp. 162169, Sept. 2018

[2] X. Tan, Z. Sun, D. Koutsonikolas, and J. M. Jornet, "Enabling indoor mobile millimeter-wave networks based on smart reflect-arrays," in Proc. IEEE InfocomINFOCOM, Apr. 2018, pp. 270-278.

[3] L. Li, T. J. Cui, W. Ji, S. Liu, J. Ding, X. Wan, Y. B. Li, M. Jiang, C.-W. Qiu, and S. Zhang, "Electromagnetic reprogrammable codingmetasurface holograms," Nature Commun., vol. 8, no. 1, p. 197, 2017.

[4] Y. Han, W. Tang, S. Jin, C. Wen, and X. Ma, "Large intelligent surfaceassisted wireless communication exploiting statistical CSI," IEEE Trans. Veh. Technol., vol. 68, no. 8, pp. 8238-8242, 2019.

[5] E. Basar, M. Di Renzo, J. De Rosny, M. Debbah, M. Alouini and R. Zhang, "Wireless communications through reconfigurable intelligent surfaces," IEEE Access, vol. 7, pp. 116753-116773, Aug. 2019.

[6] Y. Han, W. Tang, S. Jin, C. Wen and X. Ma, "Large intelligent surfaceassisted wireless communication exploiting statistical CSI," IEEE Trans. Veh. Technol., vol. 68, no. 8, pp. 8238-8242, Aug. 2019.

[7] Z. Zhou, X. Chen, and B. Gu, "Multi-scale dynamic allocation of licensed and unlicensed spectrum in software-defined HetNets," IEEE Network, vol. 33, no. 4, pp. 9-15, Jul./Aug. 2019.

[8] M. Liu, J. Yang, T. Song, J. Hu, and G. Gui, "Deep learning-inspired message passing algorithm for efficient resource allocation in cognitive radio networks," IEEE Trans. Veh. Technol., vol. 69, no. 1, pp. 641-653, Jan. 2019

[9] Z. Zhou, Y. Guo, Y. He, X. Zhao, and W. M. Bazzi, "Access control and resource allocation for M2M communications in industrial automation," IEEE Trans. Indus. Inform., vol. 15, no. 5, pp. 3093-3103, Mar. 2019.

[10] M. Liu, T. Song, G. Gui, J. Hu, and H. Sari, "Deep cognitive perspective: resource allocation for NOMA based heterogeneous IoT with imperfect SIC," IEEE Internet Things J., vol. 6, no. 2, pp. 2885-2894, Apr. 2019.

[11] Z. Chu, W. Hao, P. Xiao and J. Shi, "Intelligent reflecting surface aided multi-antenna secure transmission," IEEE Wireless Commun. Lett., in press, doi: 10.1109/LWC.2019.2943559, Sept. 2019

[12] M. Cui, G. Zhang and R. Zhang, "Secure wireless communication via intelligent reflecting surface," IEEE Wireless Commun. Lett., vol. 8, no. 5, pp. 1410-1414, Oct. 2019.

[13] J. Chen, Y. Liang, Y. Pei and H. Guo, "Intelligent reflecting surface: a programmable wireless environment for physical layer security," IEEE Access, vol. 7, pp. 82599-82612, Jun. 2019.

[14] H. Shen, W. Xu, S. Gong, Z. He and C. Zhao, "Secrecy rate maximization for intelligent reflecting surface assisted multi-antenna communications," IEEE Commun. Lett., vol. 23, no. 9, pp. 1488-1492, Sept. 2019.

[15] X. Yu, D. Xu and R. Schober, "MISO wireless communication systems via intelligent reflecting surfaces," in Proc. IEEE/CIC ICCC, Changchun, China, 2019, pp. 735-740.

[16] C. Huang, A. Zappone, G. C. Alexandropoulos, M. Debbah and C. Yuen, "Reconfigurable intelligent surfaces for energy efficiency in wireless communication," IEEE Trans. Wireless Commun., vol. 18, no. 8, pp. 4157-4170, Aug. 2019.

[17] Q. Wu and R. Zhang, "Intelligent reflecting surface enhanced wireless network via joint active and passive beamforming," IEEE Trans. Wireless Commun., vol. 18, no. 11, pp. 5394-5409, Nov. 2019.

[18] W. Chen, X. Ma, Z. Li and N. Kuang, "Sum-rate maximization for intelligent reflecting surface based terahertz communication systems," in Proc. IEEE/CIC ICCC Workshops, Changchun, China, 2019, pp. 153157.

[19] Q. Wu and R. Zhang, "Joint active and passive beamforming optimization for intelligent reflecting surface assisted SWIPT under QoS constraints," arXiv preprint arXiv:1910.06220, 2019.

[20] C. Pan, H. Ren, K. Wang, M. Elkashlan, A. Nallanathan, J. Z. Wang, L. Hanzo, "Intelligent reflecting surface aided MIMO broadcasting for simultaneous wireless information and power transfer," arXiv preprint arXiv: 1908.04863, 2019.

[21] G. Yang, X. Xu, and Y.-C. Liang, "Intelligent reflecting surface assisted non-orthogonal multiple access," arXiv preprint arXiv:1907.03133, 2019.

[22] X. Mu, Y. Liu, L. Guo, J. Lin, and N. A.-Dhahir, "Exploiting intelligent reflecting surfaces in multi-antenna aided NOMA systems," arXiv preprint arXiv:1910.13636, 2019.

[23] M. Fu, Y. Zhou, and Y. Shi, "Intelligent reflecting surface for downlink non-orthogonal multiple access networks," arXiv preprint arXiv: 1906.09434, 2019.

[24] A. Damnjanovic, J. Montojo, Y. Wei, et al., "A survey on 3GPP heterogeneous networks," IEEE Wireless Commun., vol. 18, no. 3, pp. 10-21, Jun. 2011
[25] Y. S. Soh, T. Q. S. Quek, M. Kountouris and H. Shin, "Energy efficient heterogeneous cellular networks," IEEE J. Sel. Areas Commun., vol. 31, no. 5, pp. 840-850, May 2013.

[26] Y. Xu, G. Li, Y. Yang, M. Liu and G. Gui, "Robust resource allocation and power splitting in SWIPT enabled heterogeneous networks: a robust minimax approach," IEEE Internet of Things J., doi: 10.1109/JIOT.2019.2941897, Sept. 2019.

[27] H. Zhang, C. Jiang, X. Mao and H. Chen, "Interference-limited resource optimization in cognitive femtocells with fairness and imperfect spectrum sensing," IEEE Trans. Veh. Technol., vol. 65, no. 3, pp. 17611771, Mar. 2016.

[28] N. Zhao, Y. Liang, D. Niyato, Y. Pei, M. Wu and Y. Jiang, "Deep reinforcement learning for user association and resource allocation in heterogeneous cellular networks," IEEE Trans. Wireless Commun., vol. 18 , no. 11 , pp. 5141-5152, Nov. 2019.

[29] W. Dinkelbach, "On nonlinear fractional programming," Management Science, vol. 13, pp. 492-498, Mar. 1967.

[30] M. Peng, C. Wang, J. Li, H. Xiang and V. Lau, "Recent advances in underlay heterogeneous networks: interference control, resource allocation, and self-organization," IEEE Commun. Sur. \& Tutors., vol. 17, no. 2, pp. 700-729, 2015

[31] H. Zhang, C. Jiang, N. C. Beaulieu, X. Chu, X. Wen and M. Tao, "Resource allocation in spectrum-sharing OFDMA femtocells with heterogeneous services," IEEE Trans. Commun., vol. 62, no. 7, pp. 2366-2377, Jul. 2014

[32] J. Papandriopoulos and J. S. Evans, "SCALE: a low-complexity distributed protocol for spectrum balancing in multiuser DSL networks," IEEE Trans. Inf. Theory, vol. 55, no. 8, pp. 3711-3724, Aug. 2009.

[33] J. F. Sturm, "Using sedumi 1.02, a matlab toolbox for optimization over symmetric cones," Optimization methods \& software, vol. 11, no. 1, pp. 625-653, 1999.

[34] J. Lofberg, "A toolbox for modeling and optimization in MATLAB," in Proc. CACSD Conf., Taipei, Taiwan, 2004.

[35] A. M.-C. So, J. Zhang, and Y. Ye, "On approximating complex quadratic optimization problems via semidefinite programming relaxations," Math. Programm., vol. 110, no. 1, pp. 93-110, Jun. 2007.

[36] H. Zhang, S. Huang, C. Jiang, K. Long, V. C. M. Leung and H. V. Poor, "Energy efficient user association and power allocation in millimeterwave-based ultra dense networks with energy harvesting base stations," IEEE J. Sel. Areas Commun., vol. 35, no. 9, pp. 1936-1947, Sept. 2017.

[37] S. Boyd and L. Vandenberghe, Convex optimization. Cambridge, U.K.: Cambridge Univ. Press, 2004.

[38] D. P. Palomar and Mung Chiang, "A tutorial on decomposition methods for network utility maximization," IEEE J. Sel. Areas Commun., vol. 24 no. 8, pp. 1439-1451, Aug. 2006.

[39] Y. Xu and G. Li, "Optimal and robust interference efficiency maximization for multicell heterogeneous networks," IEEE Access, vol. 7, no. 1, pp. 102406-102416, Jul. 2019.

[40] K. Davaslioglu, C. C. Coskun and E. Ayanoglu, "Energy-efficient resource allocation for fractional frequency reuse in heterogeneous networks," IEEE Trans. Wireless Commun., vol. 14, no. 10, pp. 5484 5497, Oct. 2015 